\title{
Los Niveles Teóricos y Metodológicos en la Investigación Educativa
}

\author{
Theoretical and Methodological LeVels in Educational Research \\ Dr. Emilio Ortiz (eortiz@ict.uho.edu.cu) Centro de Estudios sobre Ciencias de la Educación Superior, \\ Universidad de Holguín (Holguín, Cuba)
}

\begin{abstract}
Within the current theoretical and methodological conceptions that approach the educational processes and phenomena there are several levels that, with a systemic character, offer coherence and unity from their more general argumentation to the description of how they should be studied. However, this reality, that is implicit in the different theories, is unnoticed by the researchers and thus it brings about theoretical and methodological in-consequences in the investigation which damage the strictness of the research process. The aim of this article is to analyse each level in the theoretical foundation of investigations about the educational process. A deep analysis of the various ways in which the state of the art has been approached by different authors from the theoretical and methodological points of view is made and the importance of each of one is analysed with new elements that can be enrich according to criterion of the author of this article.
\end{abstract}

Key words: theoretical-methodological levels, educational research, multilevel research, research methods, techniques.

\section{Resumen}

Dentro de las concepciones teóricas y metodológicas actuales que abordan los procesos y fenómenos educativos, existen varios niveles que con carácter de sistema les brindan coherencia y unidad, desde su argumentación más general hasta la prescripción de cómo deben ser estudiados. Sin embargo, esta realidad implícita en las teorías con frecuencia es inadvertida por los investigadores, provocando inconsecuencias teóricas y metodológicas en las investigaciones que lesionan la severidad del proceso investigativo. El objetivo de este artículo es analizar los niveles en la fundamentación teórica de las investigaciones sobre el proceso educativo. Se valora el estado del arte acerca de cómo han sido abordados los niveles teóricos y metodológicos por parte de diferentes autores y posteriormente, se precisa cada uno, destacando nuevos elementos que, a juicio del autor, pueden enriquecerlos.

Palabras clave: niveles teórico-metodológicos, investigación educativa, investigación multinivel, métodos de investigación, técnicas

\section{Introducción}

Los niveles constituyen la jerarquía, el rango, la organización y el orden lógico deductivo que existe dentro de toda concepción teórica y metodológica en la cual están presentes categorías y principios generales y específicos, como reflejo esencial de la realidad que le confiere a la teoría sistematicidad, coherencia y unidad. Mientras mejor explicitados estén los niveles, más completa, sólida y mejor argumentada será dicha concepción teórica. 
La concepción teórica-metodológica es sinónimo de teoría, es decir, un sistema de saber generalizado que explica científicamente la realidad, una elaboración mental de carácter orgánico, con una estructura interna compleja integrada por categorías, principios y leyes para interpretar su esencia, que emana de la actividad cognoscitiva (científica) y que encuentra en la práctica su criterio de verdad y aplicabilidad en el proceso de transformación de la realidad. Está íntimamente relacionada con la concepción filosófica del científico que la elabora y las condiciones sociohistóricas, espaciales y el campo de la ciencia en que se produce.

La teoría es la construcción de un sistema de representación capaz de articular diferentes categorías entre sí y de generar inteligibilidad sobre lo que se pretende conocer en la investigación científica. No son sistemas estáticos a los que se debe asimilar todo lo nuevo, sino sistemas abiertos en relación con los cuales los investigadores deben cultivar una conciencia de parcialidad, de desarrollo, y no de resultado final, como frecuentemente ocurre en la ciencia. Ellas existen en el pensamiento y la reflexión de los investigadores, sin lo cual la teoría se convierte en un conjunto de categorías estáticas y naturalizadas, que impide el contacto con los problemas al investigador.

La investigación en el campo educativo posee un arsenal importante de concepciones teóricas y metodológicas que le sirven de sustento para la fundamentación científica de los temas de pesquisa, así como para el descubrimiento de nuevos conocimientos, a partir de la obtención de datos y hechos originales y su correspondiente interpretación. Sin embargo, no siempre los investigadores son conscientes de la existencia de los niveles dentro de la teoría y de los métodos investigativos aplicados, sean teóricos o empíricos, lo que puede provocar una confusión epistemológica, con el consiguiente eclecticismo por ignorancia al mezclar incoherentemente argumentos de diferentes grados de generalidad, que lacera la rigurosidad del proceso indagatorio y debilita la cientificidad de los resultados.

El objetivo de este artículo es analizar cada nivel en la fundamentación teórica de las investigaciones sobre el proceso educativo.

Varios autores han destacado la existencia de niveles en las concepciones teóricas, como por ejemplo, Kuprian (1978), quien establece diferencias esenciales en cuanto a la definición de método científico, metódica y metodología en la investigación. Considera que la teoría tiene varias funciones metodológicas de orientar y canalizar los esfuerzos del investigador hacia la selección de los hechos y la formulación de la hipótesis, la previsión científica y la clasificación. Andreieva (1984) se refiere también a la existencia de distintos niveles metodológicos del enfoque científico como procedimiento general y particular a la vez. González (2007) se refiere a la existencia en la teoría de dos niveles estrechamente relacionados entre sí, un nivel macro, que organiza representaciones de una cierta estabilidad y alcance, que no se diluyen de forma inmediata en lo empírico, y un nivel local que, comprometido de forma más inmediata con lo empírico, va generando sus propias representaciones y conceptos, y ganando una inteligibilidad que le permite confrontarse con el nivel macro. Machado (2006) y Machado y Montes de Oca (2008) destacan que el método científico posee tres niveles en las investigaciones educativas: filosófico, cienciológico y metodológico. Por tanto, se puede afirmar que desde el siglo XX hasta el presente, algunos autores se han referido de manera explícita a los niveles teóricos y metodológicos, ya sea en investigaciones concretas o en reflexiones teóricas. Sin embargo, por su complejidad, relevancia y riqueza todavía constituye un tema insuficientemente estudiado y poco aplicado en la realización de investigaciones educativas, lo que ha provocado debilidad e inconsecuencias en las posiciones teóricas y metodológicas asumidas por los investigadores.

\section{Los niveles en la teoría}

Por tanto, los autores antes mencionados coinciden al aceptar la existencia de diferentes niveles en toda concepción teórica, los cuales subsisten de manera latente o explícita, pero totalmente discernibles mediante un proceso de abstracción teórica. A partir de estos antecedentes se proponen los siguientes niveles que integran los aportes anteriores, pero de una forma más sistemática: 
Nivel teórico-general

Incluye a los referentes filosóficos y epistemológicos que le sirven de base a la concepción teórica e incluye la metodología general del conocimiento científico, los supuestos ontológicos y epistemológicos, los cuales el investigador debe conocerlos esencialmente como parte de su cultura científica y tenerlos en cuenta, de acuerdo con la teoría asumida, para mantener la imprescindible coherencia y consistencia que evite posiciones eclécticas. Por ejemplo, dentro de este nivel estarían los referentes positivistas, neopositivistas, fenomenológicos o lógico-dialécticos que están presentes en las concepciones teóricas contemporáneas.

Nivel teórico-particular

Referido a la conceptualización teórica de la disciplina científica específica con sus principios, leyes, categorías y conceptos propios como sistema en su coherencia lógica jerarquizada, como reflejo de sus relaciones esenciales y consistencia externa con respecto a otras concepciones. Tiene como antecedente el nivel anterior y es consecuente con él, constituye su derivación lógica en una ciencia particular. Por ejemplo, en este nivel están las concepciones conductistas, piagetianas, vigotskianas, cognitivistas y constructivistas, que coexisten en la actualidad y son más fácilmente identificables que el nivel teórico-general por su carácter evidente y no latente.

Nivel metodológico

El conjunto de métodos generales y particulares, teóricos y empíricos, incluyendo a las técnicas y procedimientos para investigar un objeto. Se encuentra en plena correspondencia con los niveles precedentes, pero con una independencia relativa porque si bien es cierto que las concepciones teóricas predominantes privilegian determinado arsenal metodológico, no quedan en la práctica investigativa ligados obligatoriamente a ellas porque pueden aplicarse en otras sin desmerecer el rigor científico. Por ejemplo, el método experimental, con sus referentes cuantitativos propios del enfoque positivista en las investigaciones de corte conductista en educación, es totalmente viable aplicarlo en una investigación que no tenga de base esta concepción teórica general. De igual modo, el método hermenéutico, con su origen en el enfoque cualitativo, es lícito utilizarlo en otras investigaciones que respondan obligatoriamente a otro enfoque.

Nivel sociológico

Referido al impacto social que ha tenido la concepción teórica particular mediante su aplicación continuada y sistemática en la práctica profesional e investigativa dentro de un contexto histórico-social específico. Las teorías científicas están condicionadas por la época en que surgen y que le confiere su pertinencia y justificación, a partir de los problemas sociales imposibles de resolver científicamente hasta ese momento. El efecto, las transformaciones y modificaciones provocadas por los nuevos conocimientos científicos deben dejar su impronta de manera que promuevan el desarrollo social, de lo contrario no resistirían la prueba del tiempo y dejarían de existir. El carácter social de la ciencia en la contemporaneidad constituye en basamento esencial de este nivel.

Entre estos niveles hay una relación dialéctica porque reflejan la contradicción independencia-dependencia, ya que cada uno posee identidad propia que la distingue de los demás y le confiere cierta libertad, pero a la vez existe una complementariedad al manifestarse una subordinación interna por derivarse uno del otro desde una lógica deductiva. También se refleja la contradicción general-particular, al ser cada uno más abarcador que el subsiguiente y este a su vez, más específico que el anterior.

Los niveles en las teorías científicas son una manifestación del carácter de sistema que toda concepción debe tener en su intento de reflejar la realidad en sus elementos más esenciales, desde las posiciones más generales hasta las prescripciones de cómo estudiar rigurosamente su objeto y su correspondiente impacto social. 
Las concepciones teóricas relevantes en el campo educativo han tenido en las condiciones histórico-sociales en que emergieron un efecto significativo en la sociedad, pues su surgimiento y desarrollo ha estado condicionado socialmente. Las concepciones clásicas, por haber trascendido a su tiempo, han influido en el desarrollo de la educación, como por ejemplo, la Concepción Neoconductista de Skinner, las concepciones humanistas de Rogers, la Escuela de Epistemología Genética de Piaget y la Escuela Histórico Cultural de Vigotsky. Todas surgieron en el siglo XX y su influencia se ha mantenido hasta hoy con el enriquecimiento incesante aportado por sus fieles y brillantes seguidores, así como con un impacto social significativo en el desarrollo de la educación, con sus comprensibles diferencias en los diferentes países en que dejaron su impronta.

La delimitación de cuatro niveles de análisis en las teorías científicas permite una valoración más integral y sistemática de toda teoría científica, ya que revela y delimita sus contenidos esenciales y relacionales en una lógica formal y dialéctica. Cuando se desea valorar rigurosamente cualquier concepción teórica, estos niveles devienen en una herramienta útil, aunque la independencia relativa de cada nivel concede al investigador la libertad de no tener que abordarlos explícitamente todos, de acuerdo con los objetivos de su investigación, pero él sí debe conocerlos previamente como parte de su cultura científica, sobre la base de un concienzudo estudio histórico social de su surgimiento y evolución.

Una manifestación expedita de madurez en una concepción teórica es la nitidez que logra en la delimitación precisa y coherente de sus niveles, teniendo siempre presente que es un proceso perenne porque la teoría se encuentra siempre en constante desarrollo, a partir del enriquecimiento que aportan las investigaciones, las cuales deben promover la potenciación del cuarto nivel referido al impacto científico y social. Cuando se logran revelar y fundamentar nuevos elementos teóricos en cualquiera de los niveles, que no hayan sido destacados hasta ese momento y que contribuyan a enriquecerlos, deviene en un aporte teórico válido en el proceso investigativo al enriquecer el sistema de conocimientos científicos.

Pero los niveles no solo existen en la teoría, sino también en la metodología.

\section{Los niveles en la metodología}

En las definiciones de metodología aportadas por Kuprian (1978) y Andreieva (1984), abordadas anteriormente, se manifiestan los niveles desde lo teórico general hasta lo particular. Además, un nivel importante en las teorías es el metodológico, pero no basta con declarar su presencia, es imprescindible analizarlo detenidamente para precisar mejor su valor en el proceso investigativo.

Los términos metodología y método han sido utilizados de manera indistinta y hasta como sinónimos por parte de diferentes investigadores, de acuerdo con las posiciones epistemológicas asumidas por cada uno. Un componente imprescindible en los diseños investigativos es el referido a la metodología, la cual incluye, entre otros, al problema científico, la hipótesis o idea a defender, la muestra, los métodos teóricos y empíricos.

En la fundamentación del problema científico está presente el nivel teórico particular de manera manifiesta, además de las exigencias lógicas y empíricas para su acertada elaboración. En la historia de las disciplinas científicas existen múltiples ejemplos que ilustran la supeditación de los problemas investigativos a los referentes teóricos predominantes, por lo que un mismo problema ha sido examinado desde diferentes miradas.

González (2007) afirma que el problema puede convertirse en el primer momento del modelo teórico que se irá desarrollando en el curso de la investigación. Precisa que la formulación del problema constituye la primera aproximación al objeto de investigación desde las posiciones de partida del investigador y se facilita en aquellos con años de práctica profesional que a problematizarla consuetudinariamente. 
Precisamente, en la identificación y formulación del problema subyace el nivel teórico-general debido a que requiere de una postura teórica del investigador, así como también el nivel sociológico porque el ejercicio continuado de su profesión (educativa en este caso), le permite constatar su relevancia y novedad que justifica la realización de una investigación.

En lo que respecta a las hipótesis o ideas a defender también se detectan los niveles precedentes porque ellas requieren de una fundamentación teórica, además de una apoyatura lógica y empírica. En toda hipótesis hay una explicación predecible del fenómeno objeto de estudio desde una concepción teóricametodológica específica. Aquellas que intentan dilucidar una regularidad deducible y generalizada a toda la población de sujetos investigados se basan en argumentos científicos diferentes de otras que no tienen ese alcance ni lo apetecen, porque lo importante es su expresión en los sujetos muestreados sin ánimos más abarcadores, pero con resultados científicos de validez general.

El tipo de muestreo seleccionado constituye igualmente una manifestación evidente, dentro del nivel metodológico, de los anteriores. El muestreo presupone la búsqueda de una representatividad estadística con respecto a la población, que permite la utilización de técnicas paramétricas o no paramétricas en la determinación de diferencias significativas en la búsqueda de lo nomotético y generalizar los resultados científicos como propios de dicha población. El propio concepto de muestra está cargado de limitaciones epistemológicas por su origen y por tanto, el investigador debe conocerlas para evitar un uso acrítico y nocivo.

Los métodos teóricos se utilizan de manera reiterada desde la elaboración del diseño investigativo, a partir del estudio del estado del arte sobre el problema científico, hasta la interpretación de los datos y hechos constatados y las correspondientes conclusiones y recomendaciones. Los métodos empíricos incluyen a las técnicas o metódicas útiles para la obtención del material fáctico. Pero su aplicación continuada no garantiza que los investigadores logren una congruencia interna entre ellos, ya que se conciben erróneamente como independientes entre sí, lo que fomenta cierto esquematismo en su utilización e inconsecuencias entre la concepción teórica admitida y los métodos empleados. González (2007) se refiere a esta distorsión con el nombre de metodologismo, donde los instrumentos y las técnicas se emancipan de las representaciones teóricas y se convierten en principios absolutos de legitimidad para la información producida por ellos.

Los métodos teóricos en toda investigación educativa exigen una cabal correspondencia con los dos primeros niveles de la teoría que los precede, de lo cual debe preocuparse y ocuparse todo investigador. Sin embargo, no siempre ha acontecido así pues usualmente se declaran en el diseño, limitándose a la definición de cada uno sin detallar cómo fue aplicado, o sea, su utilidad. Por ejemplo, los métodos de la unidad de lo histórico y lo lógico, de la ascensión de lo abstracto a lo concreto y de la unidad del análisis y la síntesis, son muy empleados en las investigaciones educativas, lo cual es pertinente y atinado. Pero son a la vez principios de la lógica dialéctica y su asunción posibilita que la fundamentación teórica se corresponda con concepciones que hacen de la dialéctica su piedra de toque, así como la posterior interpretación teórica de los datos y hechos científicos investigados.

Otro método teórico declarado con frecuencia es el sistémico, el cual da por sentado que la concepción teórica asumida cumple explícitamente con este enfoque y que sus exigencias sean cumplidas estrictamente durante todo el proceso investigativo hasta en el aporte científico. Por su parte, el método hipotético deductivo, como su nombre lo explicita, condiciona la aceptación de una epistemología en la que el saber teórico se produce de lo general a lo particular, lo cual entraría en franca oposición con cualquier metodología inductiva, que a través de sus métodos y técnicas empíricas intente seguir el camino contrario: de lo particular a lo general.

La modelación como método teórico es muy empleada también. Es un esquema teórico de un sistema o realidad compleja que se elabora para facilitar su comprensión y el estudio de su comportamiento, aportando los modelos como representaciones ideales de objetos reales que constituyen un eslabón 
intermedio entre la realidad y los investigadores. González (2007) define a los modelos como la construcción de una representación teórica que se mantiene en constante desarrollo ante la emergencia de nuevas informaciones empíricas. Son una forma peculiar de abstracción en que las relaciones esenciales del objeto están fijadas en enlaces y relaciones visualmente perceptibles y representadas de elementos materiales o semióticos (Davídov 1988).

Un modelo es mucho más sencillo que una teoría en su alcance y pretensiones, sin embargo, puede estar en la génesis de una nueva teoría, cuando el diálogo entre el modelo y la teoría general de la que parte el investigador se torna imposible. Los modelos son una forma de saber local que progresivamente puede conducir a una teoría general sobre procesos que trascienden los marcos que estimularon la creación del modelo.

Por tanto, en el contenido de todo nuevo modelo educativo, como resultado de aplicar el método teórico de la modelación, se expresa el nivel teórico particular y contribuyen a enriquecer a la propia teoría que le sirvió de sustento, lo que muestra también la manifestación del nivel metodológico porque ofrece una vía, un camino en el perfeccionamiento del proceso formativo, así como también el nivel sociológico por el impacto posterior que tendría mediante su introducción y posterior generalización en el campo educativo.

Por ejemplo, en una investigación educativa que aporta desde el punto de vista teórico un modelo didáctico para perfeccionar el proceso de enseñanza-aprendizaje de una asignatura específica en el nivel universitario, requiere que el investigador se apoye en una concepción teórica determinada (nivel teórico particular), y a la vez propone vías didácticas novedosas que devienen en herramientas prácticas para que el profesor perfeccione dicho proceso (nivel metodológico), promoviendo una elevación en su eficacia al ser utilizado de manera masiva por el claustro de docentes (nivel sociológico). Pero también el carácter novedoso del modelo didáctico entra a formar parte del arsenal de esa concepción teórica enriqueciéndola.

En los métodos empíricos aplicados se manifiesta también esa concordancia entre los niveles porque los datos y hechos científicos obtenidos deben ser interpretados, abstraídos y generalizados desde el primer nivel de la metodología, precisados previamente desde la fundamentación teórica y en los métodos teóricos.

De manera general se asume que el experimento y la observación son los métodos empíricos más aceptados en toda investigación. Pero el tipo de experimento (natural, de laboratorio, cuasiexperimento, etc.) que se aplique debe adecuarse a los niveles anteriores, pues la concepción que subyace en su diseño y ejecución lo matiza y determina. Igualmente, el tipo de observación elegida (participante o no participante) siempre deberá concordar con sus presupuestos teóricos. Con relación a las llamadas técnicas o metódicas de investigación por su nivel de especificidad, si bien son independientes de una concepción teórica particular, la contienen en su elaboración, aplicación, análisis e interpretación de los datos y hechos que aportan el nivel teórico-general y el particular. Por ejemplo, las encuestas y las entrevistas, muy utilizadas en las investigaciones educativas son diseñadas, aplicadas, analizadas y explicadas de acuerdo con las posiciones teóricas sustentas por los investigadores, por lo que serán más o menos formalizadas, abiertas o cerradas, estructuradas o semiestructuradas, con un tratamiento estadístico riguroso o elemental, en dependencia de cómo se desean obtener los datos y hechos científicos.

Otras técnicas investigativas muy empleadas también, que reflejan de manera más expedita los referentes teóricos de partida, son el cuestionario, la composición, el completamiento de frases y el estudio de casos porque aportan una riqueza de contenido en sus datos que es difícil de legitimar de otra forma que no sea desde posiciones ideográficas, es decir, específicas por no haber intenciones de generalizar al ser las respuestas engendradas por los sujetos investigados valiosas en sí mismas como singularidades y no porque se lleguen a reiterar como regularidad (posición nomotética). 
Las técnicas estadísticas han tenido un papel relevante en las investigaciones educativas, como resultado del enfoque positivista en general y de la influencia neoconductista en particular. Pero con el rechazo a estas ascendencias, se negó también de manera infundada el aporte valioso de estos procedimientos matemáticos en la agrupación y análisis de los datos. La estadística no está reñida con la epistemología cualitativa porque las categorías dialécticas cantidad-cantidad son inseparables (se presuponen sin ser identificables). La dialéctica de lo empírico y lo teórico se revela en este caso porque lo cuantitativo es un momento de lo cualitativo aunque son contrarios, o sea, que la cualidad, como determinación interna de un fenómeno, puede ser expresada numéricamente y para el investigador es útil.

La propia identidad relativa del nivel metodológico en las concepciones teóricas permite la no dependencia absoluta de las técnicas derivadas y este es el caso de los diseños estadísticos, los cuales son imprescindibles cuando se aplican métodos empíricos, pues ellos no son más que instrumentos que coadyuvan, facilitan y le confieren a todo proceso investigativo el imprescindible orden, organización y rigor de todo esfuerzo por aprehender la realidad por vía científica.

La existencia de una variedad importante de técnicas estadísticas descriptivas e inferenciales con el soporte moderno y cómodo de aplicaciones informáticas diseñadas expresamente para su uso, resultan de un apoyo inmenso a los investigadores y su rechazo por desconocimiento o subestimación constituyen una manifestación evidente de incultura científica de aquellos que se parapetan en pretendidas posiciones críticas hacia la tendencia empirista, que sobredimensiona la estadística en los diseños investigativos.

En toda investigación sobre problemas educativos que no sea en el plano teórico y que aplique métodos empíricos, se debe emplear la estadística mediante la conformación de tablas con sus correspondientes gráficos, y así componer la información obtenida para su análisis e interpretación de acuerdo con las posiciones teóricas de partida. Esta información resulta tan valiosa que es pertinente su conservación futura por la riqueza que contiene y su posible uso como medio de contrastación en estudios longitudinales.

Precisamente, la triangulación solo es posible lograrla de manera convincente si los datos son previamente dispuestos estadísticamente para poder compararlos, aunque sea en un plano cualitativo, y así revelar las coincidencias, reiteraciones y posibles regularidades.

Como bien plantea González (2007), la cuantificación es totalmente legítima en la producción del conocimiento, el problema está en lo que se cuantifica, es decir, dentro de qué sistema teórico toma significado, porque la significación de cada registro empírico durante el desarrollo de un sistema teórico es, necesariamente, un acto de producción teórica, pues es inseparable del sistema teórico que en su conjunto está por detrás de ese acto de inteligibilidad.

Por tanto, en la metodología los niveles están implícitos, hay que reconocerlos explícitamente y ser consecuentes con ellos para acatar la coherencia y rigurosidad del proceso inquisitorio, ya que como bien plantea Bonilla et. al. (2009), el método es técnico, epistémico y teórico.

Hasta aquí se ha argumentado a favor de la existencia de los niveles en la concepción teórica y en los métodos y técnicas aplicadas, con el apoyo bibliográfico de varios autores que se han pronunciado al respecto, pero también es posible encontrar criterios acerca de su presencia en el contenido que se investiga, es decir, en el objeto de estudio.

\section{Los niveles en el objeto de estudio}

En las ciencias sociales contemporáneas, desde el siglo anterior, se han venido desarrollando la denominada investigación multinivel, las cuales abordan objetos de estudio que se encuentran en diferente orden referidos a conceptos de jerarquías distintas, con el objetivo de aportar resultados científicos más abarcadores en el plano social. Lo cual es relevante para la investigación educativa porque contribuye a 
ofrecer una perspectiva más global y holística de la realidad estudiada, como contraposición de los estudios empírico-analíticos predominantes. En varias publicaciones iberoamericanas se encuentra el abordaje de dichas investigaciones: Mugny y Doise (1991), González y Peiró (1992), Gaviria (1992), Arróspide y Cerrato (1996) y Castro (1999).

González y Peiró (1992) destacan la perspectiva multinivel al estudiar la conducta organizacional, pues en ella participan agentes que ocupan distinto orden conceptual y de inclusión. Y se refieren a la investigación multinivel como la que tiene en cuenta variables ubicadas en más de una jerarquía. Estos autores definen la unidad focal como aquella que acapara el interés teórico del investigador e indica el nivel en el que hay que operacionalizar la variable dependiente y en el que deben realizarse las generalizaciones.

Mugny y Doise (1991) abordan los niveles de análisis en el estudio experimental de los procesos de influencia social desde la óptica de la psicología social, con el objetivo de constatar fenómenos que ocurren a nivel individual (subjetivo), como resultado de las relaciones interpersonales y grupales.

Arróspide y Cerrato (1996) se refieren a la necesidad de estudiar el grupo analizando el marco en el que vive, con varios niveles de explicación: intraindividual, interindividual o situacional (posicional) e ideológico (social y cultural).

Estas consideraciones sobre la coexistencia de varios niveles en la investigación de objetos de estudio complejos reflejan la necesidad de conocerlos más profundamente en su dificultad e interconexión interna con otros fenómenos sociales en general y específicamente en el campo educativo. La tradición investigativa en educación ha enfatizado en uno u otro nivel, lo cual ha sido una necesidad como precedente obligado del conocimiento científico al ir de lo simple a lo complejo. Si no se hubieran estudiando los niveles aislados primero fuera imposible llegar a estas indagaciones multinivel más profundas. Las cuales han aportado resultados científicos originales con el apoyo de técnicas estadísticas modernas y novedosas, que trascienden las disciplinas particulares donde fueron realizadas al servir de referentes a otras dentro de las ciencias sociales.

En las investigaciones que tienen por objeto el fenómeno educativo en su complejidad y fuerte raigambre social, es importante distinguir y delimitar explícitamente qué nivel o niveles se estudian para la determinación del alcance de los resultados científicos y sus correspondientes generalizaciones. En el proceso educativo escolarizado están presentes varios niveles de influencia social, como son el personal (subjetivo), interpersonal (intersubjetivo), el grupal y el comunitario. Cada uno está bien deslindado, definido desde el punto de vista científico y con la posibilidad de ser seleccionados como objetos de investigación independientes, aunque es imposible aislar o evitar las interacciones e interinfluencias que existen entre ellos por su sistematicidad y complejidad.

Por ejemplo, en los estudios sobre el aprendizaje escolar se ha enfatizado en el nivel personal e interpersonal, pero poco en los niveles grupal y comunitario. Sobre la formación de valores hay resultados científicos abundantes en los niveles interpersonal, grupal y comunitario, pero no en el nivel intrapersonal. Y la formación de competencias profesionales ha sido vista desde el nivel interpersonal, pero no desde los niveles intrapersonal, grupal y comunitario.

Como la investigación científica es un proceso de abstracción, cada uno de estos niveles resulta un objeto de estudio válido, teniendo siempre en cuenta las limitaciones que provoca restringirse a un solo nivel, por lo que de los resultados científicos obtenidos no se deben hacer generalizaciones que involucren a otros no investigados. Precisamente, las investigaciones multinivel tratan de solventar los impedimentos de los estudios de los niveles aislados para el logro de estudios más integrales, los cuales reflejan mejor en su complejidad y dinámica los procesos y fenómenos estudiados. 
La interdisciplinariedad, como tendencia científica predominante en las investigaciones contemporáneas, constituye un soporte oportuno y efectivo en las investigaciones multinivel, las cuales exigen de los investigadores gran preparación cultural general previa de las disciplinas científicas confluyentes, agrupados mediante proyectos de investigación con objetivos de largo alcance.

A las investigaciones multinivel se llega mediante el estudio previo de cada uno de los niveles implicados, como un proceso paulatino de acercamiento sucesivo a objetos más complejos, cuyos resultados científicos pueden ser más generalizados a la realidad y contribuir a la solución de los problemas que promovieron su búsqueda. Estos resultados científicos más generales pueden provocar la aparición de nuevos principios, categorías o conceptos más abarcadores que incluyen a otros particulares o parciales asociados a los niveles previamente investigados.

Estas investigaciones multinivel deben fundamentarse en concepciones teóricas y metodológicas de carácter interdisciplinar, con la aplicación de varios métodos y técnicas de investigación incluyendo las estadísticas, que proporcionen una gran riqueza de datos y hechos científicos, con la correspondiente triangulación para realizar entonces lo más difícil: un análisis e interpretación de ellos desde la teoría asumida, pero buscando nuevos elementos que la enriquezcan como reflejo de la complejidad del objeto que se investiga.

En las concepciones primigenias de la Escuela Histórico Cultural de Vigotsky están latentes estos niveles cuando se aborda el origen social de los procesos de psicológicos superiores, el proceso dialéctico de lo interpsicológico a lo intrapsicológico y el papel de la sociedad, de la cultura, de las demás personas en general y de los maestros y coetáneos en particular, en el desarrollo psicológico personal expresado en el concepto de zona de desarrollo próximo y situación social del desarrollo para el sujeto como individuo en su tiempo histórico (biográfico) y generacional.

\section{Conclusión}

Hasta ahora las investigaciones de estos fenómenos como objetos de estudio desarticulados por cada nivel han aportado resultados científicos apreciables e imprescindibles, pero fragmentados porque necesitan de estudios multinivel que penetren en sus esencias concurrentes y aporten conocimientos más integrales que contribuyan a resolver los problemas que afectan al perfeccionamiento del proceso educativo.

Las exigencias del desarrollo social contemporáneo determinan la realización de este tipo de investigación compleja, con la participación de variables pertenecientes a más de un nivel, con la aplicación de varios métodos y técnicas investigativas, y la consiguiente obtención de múltiples datos y hechos científicos que son necesarios analizar e interpretar concienzudamente, de acuerdo con las posiciones teóricas de partida. De lo que se infieren las dificultades en su realización, las exigencias a los investigadores que participan, la logística necesaria y el tiempo requerido.

Sin embargo, dentro de las investigaciones multinivel actual existe una tendencia empirista predominante que acentúa la utilización de técnicas estadísticas de nueva generación, las cuales son capaces de discriminar las relaciones significativas entre múltiples variables participantes. Si bien es innegable su valor y pertinencia como apoyatura metodológica en la búsqueda de nuevos conocimientos científicos, no deben ser las responsabilizadas ni las que decidan la calidad de los resultados obtenidos, ya que le corresponde a los investigadores la misión de interpretar esa realidad numérica factual y brindarle la elaboración teórica final, al elevarse por encima de lo empírico para llegar a lo teórico, en realidad lo más difícil en el proceso investigativo.

Fariñas (2005), basándose en la concepción histórico cultural, aporta las siguientes consideraciones que enriquecen los fundamentos epistemológicos de las investigaciones multinivel: a) asumir el objeto de estudio como un todo sin su desmembramiento como ha sido costumbre, b) tomar en consideración las 
peculiaridades del objeto y no solo su caracterización general y c) aceptar el tiempo como algo inherente y no externo al objeto de estudio. Se debe estudiar al objeto en su integridad y en su devenir.

En conclusión, podemos decir que los niveles teóricos y metodológicos en las investigaciones educativas tienen una existencia y relevancia indiscutibles en el descubrimiento y aporte de nuevos conocimientos científicos para perfeccionar el proceso formativo escolarizado. Su carácter latente requiere explicitarlos a través de la argumentación correspondiente en las concepciones teóricas aceptadas y en los diseños metodológicos derivados. En las investigaciones multinivel se revelan como una condición inexcusable para el logro de resultados científicos más abarcadores e integrales de la compleja realidad educativa contemporánea.

\section{Bibliografía}

Andreieva, G. M. 1984. Psicología social. Moscú: Vneshtorgizdat.

Arróspide, J.J. y Cerrato, J. 1996. La perspectiva sociocultural en el estudio del grupo. En: S. Ayestarán (ed). El grupo como construcción social. Barcelona: Plural, pp. 174-188.

Bonilla, E; Hurtado, J. y Jaramillo, C. 2009. La investigación. Aproximaciones a la construcción del conocimiento científico. México: Alfaomega.

Castro, M. 1999. Modelos multinivel aplicados al meta-análisis. Revista de Investigación Educativa 2(17): 445-453.

Davidov, V. 1988. La enseñanza escolar y el desarrollo psíquico. Moscú: Editorial Progreso.

Fariñas, G. 2005. Psicología, educación y desarrollo. La Habana: Félix Varela.

Gaviria, J. 1992. El enfoque multinivel en la evaluación de sistemas educativos. Revista de Investigación Educativa 2(17): 429-443.

González, F. 2007. Investigación cualitativa y subjetividad: los procesos de construcción de la información. México, D.F.: McGraw-Hill Interamericana.

González, V. y Peiró, J.M. 1992. Técnicas de investigación multinivel en las organizaciones. En: M. Clemente (comp). Psicología social. Métodos y técnicas de investigación. Madrid: Eudema, pp. 349-365.

Kuprian, A. P. 1978. Problemas metodológicos del experimento social. La Habana: Editorial de Ciencias Sociales.

Machado, E. 2006. Transformación-acción e investigación educativa. Obra en opción al grado científico de Doctor en Ciencias. Centro de Estudios de Ciencias de la Educación Enrique José Varona. Universidad de Camagüey, Cuba (inédita).

Machado, E. y Montes de Oca, N. 2008. Los niveles del método científico: Una polémica actual y necesaria de la investigación educativa. Revista Pedagogía Universitaria 1(13): 105-114.

Mugny, G. y Doise, W. 1991. Niveles de análisis en el estudio experimental de los procesos de influencia social. Anthropos 27: 77-100.

Recibido el 23 de diciembre de 2011

Aceptado el 8 de febrero de 2012 